\title{
Some properties of glass
}

This content has been downloaded from IOPscience. Please scroll down to see the full text. 1902 Trans. Opt. Soc. 498

(http://iopscience.iop.org/1475-4878/4/1/308)

View the table of contents for this issue, or go to the journal homepage for more

Download details:

IP Address: 192.236.36.29

This content was downloaded on 03/10/2015 at 01:55

Please note that terms and conditions apply. 
SOME PROPERTIES OF GLASS.

Paper by Mr. Walter Rosenhatn, B.A. (Cantab.), B.C.E. (Melbourne), Member of Councul.

*Read before the Optical Society on June 15th, 1903.

THE object of the present paper is to summarise in brief form. certain comparatively well-known facts concerning those physical and chemical properties of glass which are of special importance in connection with all classes of optical instruments. For the present purpose, I propose to exclude those properties particularly known as "optical" which are expressed by the constants of refraction and dispersion. These are of vital importance, but they constitute a very large subject in themselves, and for that reason demand separate treatment. The subjects with which this paper is to deal are principally colour and transparency, hardness and durability, thermal expansion and internal strain.

In the majority of its optical uses, the most essential property of glass is its transpzrency; the only exceptions to this rule are those cases where the glass is merely used to carry a reflecting film of silver or other metal-in these cases the reason for the use of glass lies in the hardness of its surface, its power of readily acquiring and retaining a brilliant polish, and-as compared with most metals-its light specific weight. Nevertheless, in the great majority of optical instruments, glass acts as a transmitter of light. Unfortunately, very little is known as to the mechanism by which transparent substances are enabled to transmit light. Modern experience of electric waves is tending to confirm the view that waves in the ether can, as a rule, traverse the space occupied by matter without suffering more than a certain retardation; that, in fact, in this sense transparency is an almost universal property of matter, with this important reservation: that for each kind of matter, the power of allowing

* Owing to the indisposition of the author, the paper was read for him by Mr. S D Chalmers, M.A 
ether wares to pass is restricted to wares of a certain limited range of length. The bodies which are ordinarily called transparent are those which have the power of transmitting waves of that particular length which is capable of affecting the eye and of producing the sensation of light. The kind of wares which a given substance is capable of transmitting, or-what comes to the same thing-the kind of wares it is capable of absorbing, depends upon the size and weight and arrangement of the molecules, and, perhaps most of all, upon the electric charges carried by them. In the simplest class of bodies--gases-we know that a substance will absorb the same kind of light as it is itself capable of emitting when hot; and, no doubt, the absorption of light by solid bodies is governed by somewhat similar principles, only that the greater complexity of the conditions has so far prevented us from tracing the details of the phenomena. There is, however, a physical constant which can be determined for every substance, known as the "specific inductive capacity," and the electro-magnetic theory of light teaches that the refractive index of a substance should be equal to the square root of this specific inductive capacity. But this is only the roughest of first approximations, and experiment does not really confirm it. In measuring specific inductive capacity in the ordinary way we are doing something analagous to measuring the tensile strength of a piece of metal by the application of a steady load; now, it is well known that in the case of the metal we shall obtain very different results if the load be applied with a sudden jerk, and still more so if it were to be applied and reversed many thousand times per second. But in comparing the electric actions which go on when a steady electric stress is applied, as in the usual method of measuring specific inductive capacity, with those which go on when a light wave of many thousand reversals per second is passing through the medium, we are as unlikely to arrive at correct conclusions as in the somewhat analogous case of the metal. We might express this in another way by saying that theory indicates that there is a very intimate connection between the behaviour of a substance as an electric insulator and the velocity with which light-waves will travel through it; but that its insulating properties must be considered not when a steady electric pressure is applied to it, but when that electric pressure undergoes as rapid a series of alternations as the vibrations of 
the light. we are considering. Somewhat on these lines, Paul Drude has formulated a very beatiful theory of optical dispersion, but I cannot go further into this fascinating sulsject here; I shall have to refer to it aguin when dealing with the question of the colour of glasses. As regurds transparency, we can say little more than that it is associated with certain properties of electric insulators; all good conductors of electricity, such as metals, are opaque to light except in the thinnest of layers.

Transparency, to light waves, however, is not a very common property of matter, and it is interesting to consider to what classes transparent bodies lelong. We find that they are either

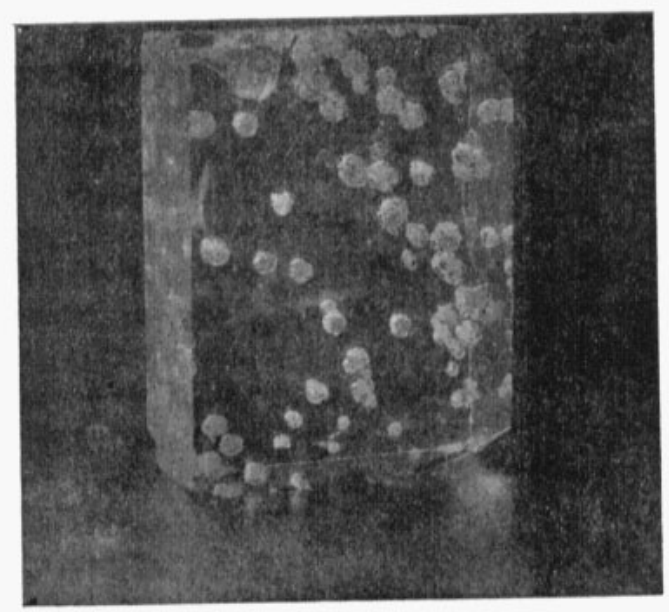

FIG. 1.

homogeneous fluids or homogeneous crystals, and the question at once arises-does glass belong to either of these classes? I have no hesitation in expressing the view that glass is not a crystalline body in its ordinary state, as it presents none of the characteristics of that class of bodies. Under certain circumstances glass may become crystanine, but its entire properties are then altered, transparency entirely disappearing. This is a phenomenon known as "devitrification," and when kept at certain critical temperatures almost any kind of glass will undergo this 
change, which I regard as the crystallising out into definite crystalline compounds of some or all of the substances present in the glass in solution or mixture. The change begins, like all crystallisations, simultaneously at a number of points throughout the mass of the glass, more or less spherolitic crystals being formed, which ultimately meet, thus occupying the entire mass. The photograph, fig. 1, shows a specimen of glass in which this process has been arrested, by sudden cooling, at an intermediate stage. The crystals in this case are Wollastonite. This property of crystallisation points to the view that glass is in reality a fluid, and owes its apparent solidity to its great viscosity. This view is borne out by several other lines of evidence, the most important of which is that glass has no definite melting or freezing point, but passes continuously from an obviously liquid into an apparently solid state, and vice versî. In a general way this fact has

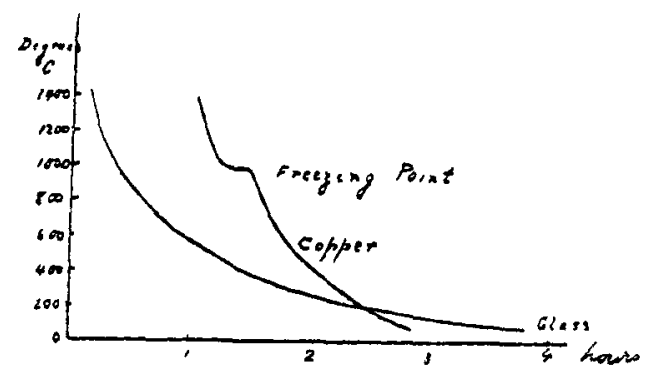

FIG. 2.

been known as long as glass itself, but I thought it worth while to apply the method used with such suceess in the study of steel and other alloys, and to take a pyrometric cooling-curre. A small crucible of glass is raised to a white heat, the thermocouple inserted and attached to a recording pyrometer, and the whole allowed to cool. The pyrometer curve represents temperature as ordinate with time as abscissa, and is reproduced in fig. 2. As will be seen, the curve is quite smooth, thus proving that no sudden solidification-or, what is the same thing, crystallisation - can have taken place. For the sake of comparison, the cooling curve of copper, with its well-marked freezing point at about $1,050 \mathrm{C}$., is also shown in fig. 2. I have gone into this subject 
somewhat fully because two English chemists have recently advanced the view that glass is a crystalline aggregate, a view I would most strongly contest. These Chemists, Messrs. Jackson and Rich,* illustrate in their paper some micro-photographs of radiating, more or less crystalline figures revealed on the surface of certain glasses by means of etching with hydrofluoric acid, but they state that these figures could only be obtained on surfaces which had been obtained by "fire polish," i.e., had solidified thus from fusion, but could not be obtained on polished sections. $t$ I am inclined to regard these figures as truly indicating the existence of rudimentary crystal-skeletons in the surface, but to attribute their presence there to an incipient devitrification, which is very apt to occur-if it does not always occur more or less-on fire-polished surfaces. Messrs. Jackson and Rich have also succeeded in separating constituents of different density from certain dense flint glasses, but this is, I think, simply a proof that the particular specimens of glass were not absolutely homogenous, which may be explained as easily by supposing an imperfect mixture of viscous fluids as a mass of mixed crystals.

Whatever the true nature of glass may be, it can only be truly trausparent when it is truly homogeneous. It is possible to see through a piece of glass only when every ray of light that enters it passes through in a straight line. In very good and perfect glass especially manufactured and selected to this end, the above condition is satisfied; but it is not by any means strictly satisfied in all ordinary kinds of glass. If we take an ordinary piece of plate-glass, we find that it is really very far from homogeneous. Looking through it in the ordinary way, it is very difficult, with the unaided eye, to detect any want of uniformity, but it becomes apparent when a greater thickness of glass is looked through. For this purpose it is only necessary to polish two opposite edges of a plate a few inches wide; it is as a rule hardly possible to see through the glass in this way at all. The defect exhibited by glass of this lind is known as "string" or "striæ," or "veins," and is no doubt due to imperfect mixing of the contents of the crucible. A very similar appearance is

* W. Jackson, A.R.C S., and E. M. Rich, B.Sc.: "The Constitution of Glass," Journ Soc. Chem. Ind 1901 555-560.

+ This result had been obtained by Leydolt in France, long ago. See Compt Rend 34, 565. 
produced whenever two miscible fluids of different density are first stirred up together; for instance, if some pure water be poured upon a dense sugar solution and stirred a little, the fluid will appear full of these strix, and they will only disappear on prolonged stirring. This defect is at once the most troublesome, frequent, and serious of all those to which glass for optical uses is liable. The veins are often so fine that even an experienced eye fails to detect them, and they are only discovered when a finished lens gives bad definition or multiple images. There are, however, methods of examining glass by which even the minutest striæ may be detected. The simplest of these is to hold the glass up between the eye and a small gas-flame, and to examine the glass through a small magnifying glass of low power, held in such a way as to be focused upon a point within the glass, which will then appear bright, any veins being readily detected as

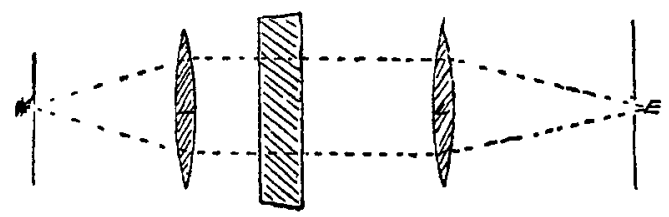

FIG. 3.

shadows. A more perfect arrangement for the same purpose is illustrated in diagram, fig. 3. Two similar simple double-convex lenses of about 6 in. focus and 3 in. diameter are set up on a common axis, about 6 in. apart. At the principal focus of one a small source of light is placed-such as a pin-hole in a screen, with a light behind it. The first lens converts the light diverging from this point into an approximately parallel beam, which the second lens converges to its own principal focus, where the eye is placed. To the eye so placed the whole surface of the nearest lens appears brightly and uniformly illuminated. If now the piece of glass to be examined be placed between the two lenses, and this glass be truly homogeneous, no change will be produced; but if the glass contain any striæ, these will at once appear as dark lines in the field, and their course can be easily marked with a suitable pencil. 
The transparency of glass is sometimes impaired by a want of homogeneity of a totally different kind, arising from the presence of foreign bodies in the mass of the glass. These may be either solid particles of opaque matter or transparent bubbles. of gas. The former defect is comparatively rare, and can be entirely avoided in all but the largest pieces of glass by suitably cutting awaJ the contaminated portions. This applies especially to those cases in which the opaque substance is a foreign body which has found its way into the glass during the process of manufacture. In some cases, howerer, this opaque material is not of foreign origin, but arises from crystallisation occurring within the glass itself. I have already referred in some detail to this process of crystallisation or devitrification, and it follows from what has been said that the facility with which it will take place will very much depend upon the chemical constitution of the glass. Now, in order to meet modern optical requirements it becomes necessary to force the composition of certain glasses until they resemble saturated, or even super-saturated, solutions, ready to deposit crystals at the least provocation; the result being in some cases the production of a very beautiful opal or porcelain.

Bubbles of gas are a very much less serious clefect, especially in optical glass, where the bubbles are small and of spherical shape, not distorted or elongated as in glass that has been blown. The only effect of the bubbles is to absorb a small proportion of light, and they do not at all interfere with the formation of the image. Moreover, in certain extreme glasses, their presence even in considerable numbers is quite unavoidable.

The transparency of glass is affected by yet another class of phenomena, viz. : those of absorption of light by the glass itself. I think it may fairly be said that the power of absorbing light is the most valuable optical property of glass. This statement is paradoxical at first sight, but is correct, because refraction. and dispersion are direct consequences of absorption : if glass did not check and absorb a small portion of the light-waves reaching it, those that passed through could not be deflected. As a rule, the glass that has the highest refractive index, i.e., which has the greatest power of deflecting light-waves, will also absorb the greatest proportion of the light that enters it. This is not strictly true, as some glasses contain substances having. 
specially absorbent properties, and also as the absorbing power of a glass is different for different regions of the spectrum, i.e., for light of different colours. Further evidence on this question of absorption is, however, needed, as determinations have only been made on a few Jena glasses for the purpose of making a selection for the lens of a large telescope at Potsdam, and these measurements revealed the fact that while flint glass showed generally a stronger absorption for all colours than crown, yet the crown glasses showed a relatively greater absorption for the red rays, while the flint glasses showed a much stronger absorption for blue and violet rays. The electro-magnetic theory of dispersion, as enunciated by Paul Drude, explains this on the assumption that for each glass there are at least two wavelengths of light-generally lying far beyond the visible range in the spectrum-for which the glass is entirely opaque, while its power of absorption increases up to this point as this wavelength is approached from either side. In certain glasses, which we call coloured, these "absorption bands" fall within the visible range; as, for instance, in a ruby glass, which only transmits red light, absorbing all the other colours. In other glasses the absorption band, although itself outside the visible range, is near to either end of the visible scale, and such a glass then shows comparatively strong absorption of light near that end of the spectrum, and its dispersion is also much greater towards that end than the other. The most marked instance of this kind is supplied by very dense flint glasses, which have a pale yellow colour, showing that their absorption-band lies near the blue end of the spectrum. Lighter flint glasses are colourless, or nearly so, when cold, but show the yellow colour when hot. Yet even in the cold, such glass when seen in large thicknesses shows a slight colour. There are a few special glasses which hardly show any colour at all even in very large pieces, but they are invariably of very low refractive index. Yet even in these glasses the existence and position of absorption bands outside the visible spectrum can be ascertained from a study of their dispersion, and can even in some cases be demonstrated. The well-known opacity of glass to long heat-waves from nonluminous bodies is an example of ultra-visual absorption. The point which is of special importance in the present connection, however, is that the colour and absorption and consequently the 
dispersion and refraction of a glass are intimately connected; that the yellow tinge of very dense flint glasses is not a defect of manufacture but inherent in the constitution of the glass, and that almost perfect transparency is only to be looked for in glasses of low refractive index. When, therefore, the transmission of as large a proportion as possible of the incident light is one of the objects aimed at in the design of a lens, the glasses should be chosen of as low refractive indices as possible, so long, of course, as other considerations do not outweigh this one.

While dealing with the subject of colour and transparency, it is necessary to refer to certain other causes of colour and absorption which are not inherent in the nature of the glass, and arise from more or less avoidable contaminations introduced into the glass by the raw materials or during the manipulations of manufacture. These contaminating substances are themselves deeply coloured, and are present in the glass either in solution or in suspension. That they do not enter deeply into the composition of the glass is shown by the fact that the effect of their presence upon the refraction and dispersion of the glass is very slight. I am, therefore, inclined to regard them as mechanical admixtures in a state of colloidal solution, and I think that Zsigmondy's recent demonstration of diffraction effects arising from the presence of fine particles in certain ruby glasses proves the correctness of that view in those cases. There is, however, one interesting point of attachment between the colours due to inherent constitution and those due to foreign colouring matter; it is this-that the extent to which a given amount of colouring substance affects the colour of a glass is greater for denser, or more highly refracting glasses than for lighter glasses. In other words, as the refractive index of a glass increases, so the glass becomes more sensitive to colouring matter. Glasses of high refractive index are therefore more liable to be coloured by minute traces of impurity than glasses of low refraction, and as such impurities are never entirely absent, it follows that where extremely white glass is desired, a glass of low refraction must be chosen.

From the practical point of view I am inclined to think that two much importance is attached to the colour of glass. A faint tinge of green can hardly affect the image in a camera, for 
instance, and even when a plate of glass appears distinctly green, the small thickness used for most lenses is hardly ever sufficient to allow the colour to be detected in the finished article. Now, in most cases, it is possible to cloak such a green colour in a glass by adding to the glass a minute quantity of a substance giving a complementary pink tinge, and thus the demand for a "colourless" glass is met. But what has really been done in such a case? The green tinge in the glass is, of course, due to the absorption of a certain proportion of the red rays, and to balance this absorption and so render the glass colourless, a substance has been introduced causing a corresponding absorpcion of blue rays, so that by requiring a glass to appear colourless we have diminished and not increased the total amount of light it transmits. I therefore feel justified in describing the demand for colourless glass, when pushed beyond a certain point, where the colour would become apparent to the eye, as a prejudice.

A further point remains to be considered in connection with the colour of glass, and that is the permanency of such colour. It is a well-established fact that certain glasses change their colour under the action of sunlight, although one finds it diffcult to believe that so stable and apparently inert a substance as glass should be liable to a class of change which we usually associate with the delicate and unstable substances dealt with by the Photographer. Evidence of colour changes in glass is, however, plentiful. In the glazed roofs of some large buildings, such as railway stations-when they are clean enough to be examined-it will be seen that some of the panes show a yellow or even a pink tinge, and there can be no doubt that originally these were of the same faintly greenish tinge as the others. Many instances of this kind are to be found in the window-panes of some of the older houses in London; a very prominent example is a window at No. 4, Marble Arch, which has a very decided pink tinge. If it be urged that in these cases the glass may have been pink to begin with, I am in a position to say that I have seen glass of known history which has undergone the same change.

A large number of experiments have been made upon this subject, those who have dealt with it including Faraday, Pelouze, Fresuel, Bontemps, Melloni and Hunt, and Gaffield. The 
latter has carried out a very elaborate series of tests of all manner of glasses on the roof of his house in Boston, some of the glasses being exposed for over 13 years.* He found changes of colour in what may be termed "white" glasses, as follows :From white to light yellow, light green to yellowish-green, brown and greenish-yellow to various tints of purple, light green to light blue; and, in some cases, merely a deepening of the original tint. In the majority of coloured glasses, he found little alteration, except in violet and brown tints. Nevertheless when ancient coloured glass is removed from its setting, it is often found that the portions of the glass which have been protected from the light are of a different tint from those portions which have been freely exposed, so that there can be little doubt that we do not now see these ancient stained glass windows with the colours they originally possessed, and it is curious to enquire how far these windows owe their matchless beauty of colour and softness of tone to the long-continued action of light.

Another and very important example of the sensitiveness of glass to light has been pointed out by Sir Benjamin Stone in connection with photographic plates; if a developed plate be cleaned off and re-coated and then again used, prints from the second negative will sometimes show traces of the image of the first negative, thus causing the mysterious appearance of "ghosts" on the photograph. Apparently some change has been caused in the glass itself by the action of light, either during exposure and development, or-in $m y$ opinion, more probably-in the printing process.

It is difficult to offer any reasonable explanation of these phenomena of photo-sensitiveness in glass. The only theory which has come to my knowledge attributes the change in colour to a change in the state of oxidation of the manganese and iron present in the glass, and evidence for this supposition is found in the fact that the pink tinge sometimes assumed by the glass resembles the pink tinges which can be imparted to glass in the first instance by the addition of a rather larger quantity of manganese in the form of peroxide. Further, all the glasses of which I have records of colour changes, contain either manganese and iron, or lead, or all three. A recent

* American Association for the Advancement of Science, Boston, 1880. 
discovery bears upon this point. It has been found that the rays from radium have the power of changing the colours of certain glasses.

For the optician, the uses of coloured glass are almost exclusively those of light-filters in photography, and I need not enter into the details of the choice of glasses for special purposes. It is obvious how conveniently the spectroscope may be used as a guide in such questions, especially when used so as to record its results photographically. Unfortunately, however, it is often very diffcult to find a glass of the right colour, and, what is equally important, of the right intensity of colour. In very thin sheets, coloured glass will often transmit rays which are entirely cut off by a greater thickness. The importance of paying attention to this point becomes evident when we consider that in a great many coloured glasses, the sheet of actual coloured substance is very thin indeed. These are the so-called "flashed glasses," where a sheet of white glass simply bears a thin film or veneer of deeplycoloured glass on the surface. Such flashed glasses may be readily distinguished from glasses coloured throughout their mass by looking through a small piece edgewise. The "flashed" glass will appear white or light green, while the solid coloured glass will appear either black or deeply-coloured with its proper colour.

In considering the changes which the colour of glass is liable to undergo, I have dealt with one class of instability to which glass is subject; I come now to deal with another class of changes which glass may undergo, viz., alterations of surface, or, rather, alterations commencing at the surface. This is a subject upon which both glassmakers, physicists and chemists have spent much time and money in research and experiment, with the result, however, that the causes and nature of these changes are now fairly thoroughly understood. I can only attempt to summarise the most important points.

The surface of glass is liable to deterioration in several ways. Of these, probably the most frequent and serious is direct physical injury, generally in the form of scratches. Those accustomed to handle finished glass are doubtless aware of the precautions requisite to avoid such injury, but I do not think that the users of spectacles, for instance, are aware of them; at all events, they frequently neglect them. For the present purpose, however, the important point is this, that glasses vary very much in their 
liability to scratching. This is due to two causes. One is that glasses differ very much in mechanical hardnẹss, some being almost as hard as quartz, while some of the heavy flint glasses are very soft indeed. It is obvious that the harder glass will in the course of inevitable friction with surrounding objects, meet a smaller number of particles capable of scratching its surface, so that with equal care, or want of care, the harder glass will preserve its surface longest.

To test the relative hardness of various glasses is a fairly simple matter, although it is by no means easy to obtain quantitative results. For comparison purposes, the simple test of scratching a series of surfaces of known hardness is sufficient, or, if it be more convenient, a sheet of the glass to be tested may be scratched with specimens of known hardness. In examining the hardness of glasses by soratching tests, it will be found that when considerable pressures are used every kind of glass will scratch every other kind more or less, and the character of the scratch must be examined by the aid of the microscope and taken into account in determining the relative hardness. No satisfactory scale of hardness for this purpose is in existence, the mineralogists' scale (Moh's) not being suitable, as most varieties of glass fall between two or three of its numbers. The establishment of a standard scale and method of testing the hardness of glass is very desirable. The scratching test alone, however, will not give true results unless the character of the surface tested be taken into account; for instance, the fire-polished surface of a piece of blown glass will have a different hardness from a polished surface of the same glass, while the state of annealing of the glass must also be taken into account. A diamond which will cut annealed sheet-glass with perfect ease will hardly touch the surface of the same glass as it comes from the blower before being annealed thoroughly.

Two other methods of testing hardness which have both been applied to glass should perhaps be mentioned here. One of these, which has been applied to a series of Jena glasses by Auerbach in a very complete manner, depends upon the observation of the area of contact between a spherical and a plane surface of the same glass under a known pressure. But, unfortunately, it is found that the hardness as determined by scratching experiments is not the same as that found by these 
indentation tests. For the Optician, however, it is the power of resisting mechanical injury that is of first importance, and mechanical injury is much more likely to be caused by scratches than by indentation under pressure. The other method of testing "hardness" is that of M. Jannetaz et Goldberg, and really consists in simultaneously exposing various samples of the glasses to be tested, along with a sample of standard hardness, to a steady grinding action, as on a rotating disc fed with emery. The results of tests made in this manner are of greater practical value than those obtained by indentation, but they do not in all cases coincide with the results of scratching tests.

Apart from durability of surface, the hardness of a glass has interest for the maker of optical instruments from the point of view of the ease and cost of cutting, grinding, and polishing. I think it will be generally admitted that the harder glass will grind and cut more slowly, but that it will take a better polish and be less liable to alterations of shape through slight unevenness of rubbing in the polishing stage, so that probably even from the point of view of cost in working, the advantage will lie with the harder glass, at all events in those cases where accurate figuring is required.

To return to the question of the durability of glass surfaces, we now come to a source of injury which lies midway between the purely physical injury due to scratching and the purely chemical injury due to weathering. Some glasses, on prolonged exposure to the air, become coated with a very thin film of decomposition products, and these are in some cases in the form of hard, sharp crystals. If a glass so coated be wiped with a dry cloth, the sharp angles of these crystals are dragged about over the surface, which becomes badly marked. As a rule, these crystalline deposits are soluble in water, so that if the glass befirst washed with water, this class of injury may be avoided. In good glasses it is only liable to arise if a lens has been put away for a long time and is then taken out and cleaned.

Before entering upon the consideration of the chemical deterioration of glass surfaces, it may be well to point out the great importance of the question. In optical instruments, the chemical deterioration of the glass surfaces may be a very serious matter. To take an example, it is evident that the surfaces of the prisms in the modern prismatic binocular should 
possess very great stability; in many cases they are inaccessible for cleaning except to the expert, and even for him it is a delicate matter. This becomes especially important where the binocular is intended for use at sea or in damp climates, so that extreme care in the choice of glass is necessary in this case. Fortunately, the requirements can be fully met, as no special demands need be made on the optical constants. The hard crown and borosilicate crown glasses commonly used for this purpose can be obtained of such a quality as to remain bright under all ordinary conditions. Examples of cases where great care in the choice of glasses from this point of view is necessary might be multiplied almost indefinitely; the outer lens of telescope object-glasses, and the lenses of eye-pieces nearest the eye, are particularly important examples.

In some of these cases, and especially in modern photographic objectives, demands are made upon the optical constants which render it very difficult, if not impossible, to comply with the conditions for great chemical stability. It is a deplorable fact that éven the great Jena firm have been compelled to abandon a large number of glasses having most desirable optical properties, because of their chemical instability. The chemical details of this subject are beyond the scope of the present paper; those interested will find further details on this and some of the other points dealt with in a book by Dr. H. Horestadt, recently translated into English-and considerably improved in the processby Professor and Miss Everett. The only point upon which I can dwell now, is that as a general rule extreme optical constants can only be obtained by means of extreme composition, and extreme composition always involves a risk of instability. It has been shown that high percentages of alkali, lead, boric, or phosphoric acid, tend to render a glass less stable chemically; and yet mathematical Opticians clamour for optical constants which can only be obtained by the use of these substances in proportions greater than the limit suggested by chemical considerations.

Another aspect of chemical instability of glass surfaces is. worthy of consideration, namely, in connection with the handling of the glass in the making of optical instruments. Cases have come under my notice where glass has been injured in this way, chiefly in the course of heating required for cementing lenses 
together. In one case flint glass was found to show minute yellow specks; when, on my suggestion, the lenses were heated over a spirit flame in place of the gas flame formerly employed, the specks no longer appeared, and there is no doubt that the defects were caused by the sulphur contents of the gas, especially as it is known that sulphurous gases will strongly attack glass when hot, especially in an oxidising atmosphere.

Having realised the importance of the chemical stability of glass surfaces, it becomes desirable to find some ready test whereby the quality of a given glass in this respect may be determined. Where fairly good glasses are concerned, it is too slow a process to keep samples and to note the time in which the first signs of weathering begin to appear. Still, as a final test, this is almost the only one available, so that it is in all cases a desirable practice to keep dated samples of all glasses used. Some caution is required, however, in the manner of keeping such samples. They should be kept free from any paper or other wrapping, laid on a shelf in a cupboard or other receptacle as free from dust as possible, and, when examined, they should only be touched at the edges, and not wiped in any way. The absence of dust is very important, because it is found that the chemical changes in glass surfaces always begin at dust particles, and it seems not at all unlikely that certain bacteria may play an important part in the disintegration of the glass. It is hardly necessary to say that all corrosive fumes should be excluded from the samples of glass, unless it is intended to test the power of the glass to resist such fumes-a test which is hardly ever required.

Prompter methods of arriving at a conclusion as to the durability of the surface of a glass are, however, available. These are-

(1) Chemical analysis.

(2) Exposure to the vapour of hydrochloric acid.

(3) Exposure to an ether-water solution of iodeosin.

The application of all of these tests requires a certain amount of laboratory equipment and an operator accustomed to chemical manipulation, and these are frequently not at the disposal of the user of optical glass. The testing of glass by such methods seems, therefore, to be an eminently suitable task for the 
National Physical Laboratory, and that institution will no doubt undertake such tests when they are required.

The first of these methods-chemical analysis-does not lend itself to treatment in the present paper. For the ordinary silicate glasses the analysis is fairly simple, and when properly performed its results are satisfactory and reliable. The data obtained by this means-thanks to the elaborate researches of Weber and Mylius and Foerster, enable us to form a good judgment on the keeping qualities of the glass. But when more complicated glasses are dealt with, the actual results of the analysis become uncertain, and even a correct statement of the composition of the glass will not give a certain guide to the stability of the surface. A modified form of partial analysis, however, whereby the solubility of the glass in water is determined, is applicable in all cases, and it may be fairly assumed that the rate at which the glass will be attacked by atmospheric agencies will be proportional to the solubility of the glass in cold water. For such determination of solubility, the glass is first reduced to a powder of standard grain by pounding it, and then passing through two sieves. A known weight of this powder is then exposed to a certain volume of water and the amount dissolved is then determined.

The second method of testing stability of surface, by exposure to the vapour of hydrochloric acid, is due to Weber. It is carried out as follows:-A slip of the glass to be tested is exposed to the vapour of hydrochloric acid for 24 hours. This can be conveniently done by pouring some concentrated acid into a porcelain basin and laying the strip or strips of glass to be tested across the top of the basin, care being taken that the acid itself does not touch the glass. A bell-jar is then placed over the whole. At the end of 24 hours, the slips of glass are removed from the basin and placed to dry under another bell. The test slips must, of course, have polished surfaces to begin with, and when the drying is complete, these surfaces will be more or less coated with crystals of chlorides derived from the action of the acid vapours on the bases of the glass. The durability of the glass surfaces is to be estimated from the density of this film of chlorides, a process obviously requiring much experience, and the use of a standard for comparison. For a chemist it would, perhaps, be more satisfactory to dissolve the 
film of chlorides in as small a quantity of water as possible and then to determine their quantity analytically.

The third method-by the use of iodeosin, is generally acknowledged as the best and most sensitive. Again, it is only a method of comparing the solubilities of glasses in water, but this time by using a very sensitive colour-reaction for the detection and estimation of the amount of alkali dissolved, and a standard for comparison must be exposed to the test reaction either at the same time as the sample to be tested, or else the standard tint must be carefully preserved for reference. The samples for this test should preferably be of the shape of thin slips, polished on all sides; they must be carefully freed from any grease or rouge adhering from the polishing process, then soaked for a short time in water, then washed in spirits until all the water is removed, and then washed in ether and finally inmersed in the test solution while still moist with ether. They are left in contact with the test solution for 24 hours, then rinsed with ether and allowed to dry. The surface of the glass will be coloured more or less pink, and the depth of the colour measures the extent to which the glass is subject to attack. The test solution is made up by saturating $100 \mathrm{c.cm}$. of ether with water, and then dissolving 0.1 gramme of iodeosin in the aqueous ether. This solution must be kept in well-stoppered bottles, which it will, of course, stain pink in time.

I have postponed the consideration of the thermal properties of glass to the end of this paper because they are not of very direct importance to the users of glass for optical purposes. In optical instruments we have rarely to deal with masses of glass lorge enough to involve risk from unequal expansion. Only where larger lenses are cemented together and the combination is liable to be exposed to tropical sunshine does a knowledge of the coefficients of expansion of the two glasses become of importance. If the glass is available in the shape of tubes, the expansion may be readily measured by the well-known method of the weight thermometer, and this might be extended to cases where only a rod or other solid piece is available by using two weight thermometers both of one kind of glass, but one of them enclosing the rod or piecé of the glass to be tested. The ordinary weight thermometer is then uscd to find the expansion of the tube grlass, and the expansion of the rod can be calculated from 
the behaviour of the weight thermometer containing it. This, however, is not a very desirable method, owing to its indirectness and probable inaccuracy. Where, therefore, the glass is only available in solid pieces, one of the modern methods for measuring expansion by the wave-length of light, such as the Abbe-Fizeau method, should be used. This, again, would seem to be, in those cases where the importance of the result justified the nieasurement, a fit task for the National Laboratory.

The relative expansions of glass and the metal in which it is held are of more frequent importance. As a rule, difficulties in this direction are met by allowing room for expansion, but, where this is not possible, the use of a nickel-steel, having the same coefficient of expansion as ordinary glass, is indicated.

The thermal properties of glass as affecting thermometers, gauge glasses and lamp chimneys, though very interesting, are

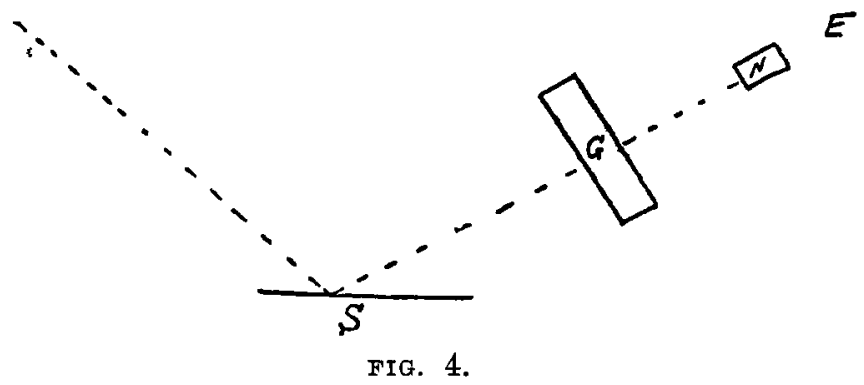

foleign to the subject of the present paper. There is, however, one way in which the thermal properties of glass do affect ontical instruments very materially, and that is by producing, under certain circumstances, severe internal strain or even fracture. Glass is an exceedingly bad conductor of heat, with the result that when one portion of a large mass of glass is suddenly heated or cooled, it expands, or contracts, long before conduction can have raised the temperature of the adjacent parts to the same degree. If this expansion is greater than the strength and elasticity of the material can withstand, fracture occurs. Consequently very great care is required in heating or cuoling glass in large masses. When glass solidifies from fusion and is allowed to cool with any sensible rapidity, the outer layers of the mass become cold and hard while the inner portions are still soft and hot. When, subsequently, the inner layers also 
cool, they are prevented from contracting by the solid outer layers, and the interior of the mass solidifies under great tension. A familiar case is the Rupert's Drop, which flies into fragments when the outer skin is ruptured. In a much milder degree such internal stress is a very common defect of glass. For many optical purposes it is of very little importance, particularly when the strain is symmetrical, as then the only effect is to slightly alter the effective refraction of the glass. In many cases, however, internal strain would practically destroy the homogeneity of the glass and render it useless, at the same time causing a serious risk of breakage when the glass is cut or ground. The defect is readily detected by the fact that strainerl glass has a polarizing effect on light. A simple method of testing a specimen of glass is indicated in fig. 4. $\mathrm{S}$ is a sheet of black glass, or a smooth board painted black, so placed that the light from a lamp or window is reflected into the eye at about the polarising angle. E indicates the position of the eye, $G$ that of the glass to be tested, while $\mathbf{N}$ is a Nicol's prism. The glass must, of course, be provided with suitable polished faces to enable the light to pass through. A strained piece of glass will show coloured patches, or even a regular pattern and cross when examined in this way. Ordinary well-annealed glass will show only a few faint shadows, while perfectly-annealed glass will show no special effects whatever. This last result is only attainable by cooling the glass at an astonishingly slow rate, but a good deal depends upon the shape of the object. Corners almost invariably cool a little faster than the bulk of the mass, thus introducing minute strains, so that where the highest degree of perfection is required, the glass should be moulded and annealed in a cylindrical shape, and the lens or block cut from that shape.

I have now endeavoured to summarise as briefly and in as practical a manner as possible some of the leading facts concerning the properties of glass with which it is of importance for the user of glass for optical purposes to be familiar; in doing so I must have repeated what is, no doubt, familiar to many of those to whom this paper is addressed, nor can this paper claim to be in any sense a complete treatise on the subject; I claim for it only the humble usefulness of a convenient resume of the leading facts. 
Professor Everetr said Mr. Rosenhain had expressed the opinion that glass was not a crystalline body in its ordinary state, and that raised the important question, What then is it? He was reminded of Graham's division of bodies into the two classes, crystalloid and colloid. Glass seemed to have a considerable resemblance to the colloid bodies, both in respect to its physical properties and also to its optical property of transparency. A very scientific suggestion had been made, and one that the speaker highly approved of, in respect to the mischief that results from trying to do good by the association of two evils which do not kill one another-the production of a "colourless" glass by the addition to greenish glass of a minute quantity of some substance giving the complementary pink tinge. It was again an interesting point that had been advanced as to the fine, rich tints seen in old stained glass windows. The suggestion that Father Time had had a hand in the production of these effects was very interesting. It was a strange fact that a diamond would hardly "touch" the surface of unannealed glass. The outer skin, Professor Everett supposed, must be in a very dense state. There must be great tension between the outer and inner layers-the inner substance (that which is the last to solidify as the glass cools) exerting a great pull. Glass, it might be mentioned, had about 15 times more resistance to "squeeze" than it had to "pull"; and in this case the strain in the skin of the glass was of the nature of a thrust, like that in a rod under longitudinal stress. A very practical suggestion had been made as to the use of nickel steel in connection with glass work. Physicists had been in the habit of saying that platinum was the only metal possessing nearly the same coefficient of expansion as glass, and available for making the metallic connections in vacuum tubes. Platinum was always made use of for this purpose, and it was said that a wire connection of any other metal would shrink away from the glass. Glass was the best material for making $x$-ray tubes; but, of course, there were objections to the use of platinum. He (the speaker) would like to ask Mr. Rosenhain if nickel steel would be a satisfactory substitute for the noble metal. Another question he would put, with reference to the colour that was so apt to appear in flint glass lenses. He had often heard that silver, present as an impurity in the lead which was one of the con- 
stituents of such glass, was the cause of the objectionable colour. Was this actually the case? It had been suggested in the lecture that the unequal refrangibility of blue and red light was due to the differences of absorption. This statement seemed very puzzling to many people. It was cleared up by reference to mechanical considerations-to the law of resonance. Let it be supposed that a pendulum is hung from a light and easilybent wooden frame. Then let a second pendulum be hung from the same frame, and suppose the first-mentioned pendulum to be set swinging. This, said Professor Everett, is the kind of action that takes place between the light waves and the molecules of glass. Those molecules which vibrate in the same time or with the same period as certain light waves, produce a suppression of that colour to which the waves in question correspond. Should the two pendulums imagined be a little out of unison there would still be an effect, the same in kind but different in degree. And just so, if there should be two places in the spectrum marked by a black absorption band-outside the visible spectrum-the absorption would strengthen as these points were approached, and the deviation of light would be increased in a corresponding degree.

Dr. Walmslet said Mr. Rosenhain had very modestly claimed, in the last paragraph of his lecture, that he had only furnished a resumé of leading facts relating to his subject, most of them familiar to those to whom the paper was addressed. He (Dr. Walmsley) could not quite admit the accuracy of that statement. It was a very valuable thing indeed to have the facts brought together as had been done in this instance. On reading the first paragraph of the paper, it might occur to one that to exclude from consideration of the properties of glass "those particularly known as optical," was something like giving the play of "Hamlet" with the part of the Prince of Denrrark left out. But it was to be hoped that Mr. Rosenhain would supplement his present paper with one discussing the particularly optical properties of glass upon some future occasion. The phenomenon of devitrification was of considerable importance, and he (the speaker) would be glad to know whether this had been observed at extremely low temperatures, such as those used in experiments with liquid air. The question of hardness must be dealt with by one of the sub-committees on 
Optical Standards; but, of course, it was necessary to have a definition of hardness in the first place. One test that had been mentioned in the paper-that of indentation under pressurewas really a test of viscosity. It was a very different matter from the test by scratching with a sharp point. It was not altogether an easy thing to decide precisely what was meant by hardness. Referring to the iodeosin reagent, it would be interesting to learn from $\mathrm{Mr}$. Rosenhain whether its indications were equally applicable in cases where glass was exposed to the corrosive action of various chemical agents different from iodeosin. Did the action of the iodeosin on glass run pari passu with the destructive influence of the other agents to which glass instruments or vessels were commonly exposed? An impression that was left by the paper to which they had just listened was, that it was rather a queer thing that glass should answer its optical purposes at all, since it was a material subject to so many different defects.

MR. HADDON asked whether devitrified glass had been investigated as to its local composition. Had those parts which solidified or crystallised out first a different composition from those solidifying out later? Was not the green tinge observed in many glasses generally due to the presence of iron? And would the lecturer state whether it was a fact that the greenish tinge produced by the presence of iron was less marked in soda glasses than in those containing potash? Nickel steel was a material varying a good deal in composition. The recently introduced nickel steel having a coefficient of expansion of one-millionth, as used for making clock pendulums and such-like purposes, would expand or contract much less than glass under the influence of changes of temperature; and it would not be satisfactory to employ this for making the cells of lenses. With regard to the use of platinum for making joints between metal and certain kinds of glass, it was generally said that platinum was the only metal available for the purpose, since it was the only one with which a leakage of gas could be prevented. Pure platinum, Mr. Haddon believed, answered better than platinum even slightly alloyed.* He (the speaker) had sealed copper wires into glass; but for this it was necessary to use a very pure copper, and then, in the opinion of the speaker, there was a certain amount of yielding of the soft metal under those stresses to which the joint 
was subjected by the influence of temperature changes. The same thing held good in the case of gold wire, which answered best for sealing into glass when not alloyed.

Mr. KENDAL having been called on to speak said that he could add little to what had already been advanced. From a practical point of view it would certainly be easy to attach too much importance to freedom from any trace of colour in glass.

Mr. Dixer said he had been very much interested in the paper read. It treated of matters on which, however, he was glad to learn but unwilling to comment. He had been pleased to hear Dr. Walmsley give expression to one of the difficulties with which they had been met. Hardness was distinct from elasticity, and from viscosity; it was the liability to be scratched. There was another very important question, as to the absorption of different kinds of light. As a spectacle maker and trader he would welcome an improved nomenclature of light rays according to their position in the spectrum-to their wave length. He would ask the scientific experts to lay down rules for the division of light according to its actinic, thermal and other properties. Those furnishing the different varieties of optical glass could then specify them in terms of their absorptiveness for the different kinds of light. The question of tints as applied to spectacle lenses-of the supposed therapeutic effects of colourwas an important one; and standardising was needed here. If we were to arrive at definite ideas on the therapeutic virtues of tinted lenses, we must have clear ideas also as to what different kinds of light are stopped by the several varieties of glass, and as to what effects these coloured lights have separately on the eye. While apologising for the vagueness of the idea, he would suggest that values should be assigned to the absolute properties of glass in terms of the properties of that light which it transmits.

Mr. Thorathwatte said it was quite true, as had been stated, that for many optical purposes little consequence attached to the thorough annealing of glass. That was the case where refraction was concerned; but in glass reflectors for optical purposes it was important that the annealing should be well done. Otherwise in the process of cooling, after leaving the tool on which the glass surface had been worked, some deformation was liable to occur-there was apt to be such an alteration of shape as 
would necessitate returning the mirror again and again to the tool. He (Mr. Thornthwaite) had made a great number of "unannealed glasses" by simply bringing the glass to about melting point in the furnace and then removing it and laying on sand to cool fairly quickly, after which beautiful polariscopic effects of colour could be obtaied. The hardness of the external skin of these specimens was such as to prevent the scratching on of a price mark with the diamond. The speaker did not think that pure silver gave any injurious chemical reaction with glass. He was delighted to see that a book written in German, dealing with the subject of optical glass, had been so admirably done into English, and made more understandable to those of us for whom the grandiloquent words of a German text-book were a little troublesome.

MR. V. H. Mackinner said that he had often observed defects on the lower lenses of the eyepieces of binoculars which had been used in a hot climate-those defects being apt to occur on that lens and that surface of the lens which was furthest from the eye. Sometimes the tree formation was so pronounced that it became necessary to repolish the damaged lens. He would be glad to know the cause of the trouble.

The President then rose to propose a vote of thanks to Messrs. Rosenhain and Chalmers. He said that he had been at first inclined a little to regret that general principles and not practical details had been discussed. However, it was to be hoped that this was only the first of a series of papers by Mr. Rosenhain dealing with the whole subject. It was to be hoped that by the enterprise of Messrs. Chance, the material would be brought together for another book like that which had just been translated by Professor and Miss Everett. This was a thing that we needed and must have in this country; and they must look to Mr. Rosenhain for it. He was the man on whom they had to depend for its accomplishment. Turning to the paper before them, the first matter dealt with was the optical defects of glass. It was the most disheartening thing he (Dr. Drysdale) knew of to find after the tedious work of calculation, and after mechanically working the surfaces of a lens, that the whole result was spoiled by the presence of striæ, which it had been impossible to discover by the ordinary method of examination in the first place. In regard to the explanation of the 
absorption bands in glass-it was known to everyone that the violet waves of light vibrated more quickly than the red waves; and flint glass being denser than crown it was natural to suppose a greater impedence being given to the particles in the former case. The denser the glass, the greater would be the check to the more rapid vibrations of light traversing it. If now it was to be inferred from what the lecturer had said that colour is a physical necessity in heavy glass, then it must be useless to experiment any further in the hope, by chemical means, of producing such glass, free from colour. But notwithstanding what Mr. Rosenhain had said, he (the speaker) would welcome a really clear glass. To improve the prism telescope, what was most necessary was to increase the angle of the cone of light; and this requirement demanded the use of glass of higher refractive index-but to retain the quality of transparency was also very important. On the question of hardness, as had been said, that quality had not yet been strictly defined; but from the practical point of view it would not be a very difficult matter to define it. Indentation tests of glass were of little practical utility, as they referred more to the elasticity of the material than anything else. Scratching was a good test, however. Mr. Rosenhain's paper bristled with interesting points. Dr. Drysdale concluded by again expressing the hope that this paper was only the preface to much more work of the same kind, which would elevate the glass industry in this country to the same dignity as it had attained in Germany. The vote of thanks which was then put to the meeting was carried by acclamation.

Mr. Rosenhain, after suitably responding to the vote of thanks, said that he would be glad to give further papers to the Optical Society when he could do so with reference to his own work. He would be sorry to give only abstracts of the work of others. Dr. Hovestadt's book, which had been referred to, was based on the researches of no less than 40 or 50 eminent scientists, who had the resources of the Reichsanstalt University laboratories at their disposal; and it would be asking a lot of him (the speaker) to expect him to give a detailed discussion of more than perhaps one or two points. Probably not more than about half a column of his paper had been occupied with really original matter. Referring to Professor Everett's 
remarks, the word "colloid" did not, he was afraid, mean much in relation to glass. He considered that glass might better be described as a fluid, and that it had not the cellulas structure characteristic of jellies. Mr. Haddon had mentioned one particular nickel steel alloy-invar-but it was quite possible by varying the proportion of alloy to get all sorts of coefficients of expansion in this material. The difficulty in fusing it into glass would be (as with all except the noble metals) the tendency to surface oxidation. If the size of the annular space that would be left between glass and metal wire of any sort, were calculated for certain varlations of temperature, it would be found to be very small indeed. He did not agree with the suggestion that had been made that the use of lead contaminated with silver was responsible for the colouring of dense flints. Silver alone would not colour glass in that way. Again, as the yellow tinge became deeper on heating the glass, it was not likely to be due to the presence of impurity-but rather, as Drude suggested, to altered electrical conditions. As to devitrification of glass at very low temperatures, he had not heard of that. Glass at the ordinary temperature of the air had been known to be practically unaltered after a thousand years. In some cases the mineralogical nature of the crystals separated out from glass could be recognised undex the microscope; but there was so much interpenetration that chemical examination was difficult. What the iodeosin test did was simply to measure the solubility of glass in water; and from this a fair deduction could be drawn as to the probable durability of the glass exposed in moist air. The difficulty with Mr. Dixey's suggestion about the classification of light waves consisted in the fact that these overlap so much in respect to their visual, chernical, or thermal properties. Glasses had not been produced, so far, which would only allow of the transmission of one kind of light very definitely. Mr. Mackinney's question could hardly be replied to without information as to the nature of the glass concerned. Perhaps the inside of the binoculars might have been varnished with a varnish containing some dyestuff like iodeosin, which would attack the glass, starting from the comparatively cold edges of the lens near to the metal, as had been indicated by Mr. Mackinney's sketch on the blackboard. By careful microscopic examination, the character of the deterioration might 
perhaps be recognised. If Dr. Drysdale wanted something to refract light very strongly and absorb it feebly, he (the speaker). thought that impossible. He remarked further that optical density does not vary with mechanical density, in a regular proportion. He regarded the electrical properties of glass as the most important to be considered in this connection. 